Nome degli autori:

Pietro Randelli, Matteo Denti, Luca Serrao, Alessandra Menon, Paolo Cabitza.

Titolo dell'articolo:

\title{
IL CONTROLLO DEL SANGUINAMENTO NELLA PROTESI TOTALE DI GINOCCHIO
}

Nome e Indirizzo completi di tutti gli Autori e loro affiliazioni e recapiti:

Dott. Pietro Randelli, MD

Dipartimento di Scienze Biomediche per la Salute, Università degli Studi di Milano U.O.

Ortopedia 2, IRCCS Policlinico San Donato

Via Morandi 30, 20097 San Donato Milanese, Milano, Italia

Tel.: 02-52.77.45.28; Fax: 02.52.77.43.12

e-mail: pietro.randelli@unimi.it

Dott. Matteo Denti, MD

IRCCS Istituto Clinico Humanitas, Rozzano, Milano, Italia

Tel.: 03-93.64.884; Fax: 03-92.20.71.63

e-mail: matteo@denti.ch.it

Dott. Luca Serrao

IRCCS Policlinico San Donato, Milano, Italia

Via Morandi 30, 20097 San Donato Milanese, Milano, Italia

Tel.: +39-345.48.05.626;

E-mail: Lucadiego.serrao@gmail.com

Dott.ssa Alessandra Menon, $\mathrm{PhD}$

IRCCS Policlinico San Donato, Milano, Italia

Via Morandi 30, 20097 San Donato Milanese, Milano, Italia

Tel.: +39-349.86.94.424;

E-mail: ale.menon@me.com

Prof. Paolo Cabitza

Dipartimento di Scienze Biomediche per la Salute, Università degli Studi di Milano, IRCCS Policlinico San Donato, Milano, Italia 
Via Morandi 30, 20097 San Donato Milanese, Milano, Italia

e-mail:paolo.cabitza@unimi.it

Corresponding author:

Dott. Pietro Randelli, MD

Dipartimento di Scienze Biomediche per la Salute, Università degli Studi di Milano U.O.

Ortopedia 2, IRCCS Policlinico San Donato

Via Morandi 30, 20097 San Donato Milanese, Milano, Italia

Tel.: 02-52.77.45.28; Fax: 02.52.77.43.12

e-mail: pietro.randelli@unimi.it

Parole chiave: sanguinamento, protesi totale di ginocchio, colle di fibrina, trasfusione.

Titolo in lingua inglese:

\section{BLOOD MANAGMENT IN TOTAL KNEE ARTHROPLASTY}

Abstract in lingua inglese:

Total knee arthroplasty is associated with high blood loss and high incidence of blood transfusion.

New drugs, as fibrin sealant, are now available to reduce these high incidence. Up to now their

effect in TKR is not clear. The aim of this study is to evaluate the effect of a new fibrin sealant, Evicel, in TKR.

Key words: blood loss, total knee replacement, fibrin sealant, transfusion 


\section{INTRODUZIONE}

La protesizzazione totale di ginocchio è una procedura chirurgica molto invasiva che può causare un'elevata perdita di sangue nel paziente durante e soprattutto dopo l'intervento. Alcuni studi hanno quantificato le perdite in un range tra 800 e $1790 \mathrm{ml}[1,2]$. Tali perdite ematiche portano ad un'importante anemizzazione che, dal 10 al 38\% dei casi, richiede una trasfusione di sangue [3]. Tale procedura, oltre a esporre il paziente al rischio di reazioni emolitiche e infezioni, aumenta le richieste alle banche di sangue, che si trovano sempre più in difficoltà a soddisfarne la domanda. La necessità, dunque, di ridurre l'incidenza di trasfusioni e i rischi per i pazienti ha portato a sviluppare diverse strategie per contenere le perdite peri-operatorie. Prima dell'intervento è possibile far depositare al paziente sacche di sangue autologhe che potranno poi essere utilizzate in caso di trasfusioni post-operatorie oppure stimolare l'eritropoiesi del paziente prima dell'intervento con eritropoietina (EPO) e ferro. Durante l'intervento i metodi più utilizzati sono sicuramente il laccio emostatico, la cementazione delle componenti (che va a tamponare meccanicamente le perdite ematiche) e l'attenta emostasi intra-operatoria con l'ausilio di elettrocauterizzatore.

Capitolo a parte nelle strategie per il controllo del sanguinamento meritano le strategie farmacologiche. Se ormai è noto che l'attività anti-fibrinolitica dell'acido tranexamico sia un'ottima soluzione, altri nuovi farmaci sono ora disponibili sul mercato, tra cui le colle di fibrina, la cui efficacia è attualmente argomento di dibattito. In particolare, tra queste nuove colle di fibrina c'è Evicel $^{\circledR}$ (Johnson \& Johnson Wound Managment, Ethicon, Somerville, NJ), un adesivo tissutale indicato per uso epilesionale come trattamento di supporto negli interventi chirurgici. Evicel ${ }^{\circledR}$ si compone di due soluzioni contenute in flaconi separati: il primo flacone contiene fibrinogeno e fibronectina umana (55-85 mg/l), mentre il secondo solo trombina umana (800-1200 IU/ml).

L'obiettivo di questo lavoro è quello di valutare l'efficacia di Evicel ${ }^{\circledR}$ nella riduzione del sanguinamento dopo artroprotesi di ginocchio.

\section{MATERIALI E METODI}

Il protocollo di questo studio controllato e randomizzato è stato approvato dal comitato etico dell'ASL milano2 (18 febbraio 2011, protocollo numero 2603) e ogni paziente ha fornito il suo previo consenso a partecipare allo studio.

Sono stati inclusi tutti i pazienti con diagnosi di osteoartrosi con valori di emoglobina (Hb) preoperatori $>11 \mathrm{~g} / \mathrm{dl}$ per le donne e $>12 \mathrm{~g} / \mathrm{dl}$ per gli uomini, senza disordini della coagulazione in anamnesi (anche conseguenti a terapie anticoagulanti o terapie antiaggreganti non sospese almeno 4 
giorni prima dell'intervento) e senza precedenti interventi sul ginocchio interessato ad eccezione delle meniscectomie.

I pazienti sono stati poi randomizzati in due gruppi: un gruppo di trattamento, a cui sarebbe stato somministrato il farmaco, e un altro di controllo, in cui non sarebbe stato utilizzato alcun placebo. La randomizzazione è stata gestita da un operatore esterno all'equipe attraverso l'uso di un software dedicato (StatsDirect Ltd, Cheshire, UK). L'equipe operatoria non è stata messa a conoscenza del gruppo di appartenenza del paziente se non al momento della somministrazione del farmaco, ossia appena prima della sutura di accesso chirurgico.

Prima dell'intervento non sono stati ammessi pre-depositi di sangue autologhi ne stimolazione dell'eritropoiesi con EPO o ferro.

La profilassi anti-trombotica per ogni paziente è stata fatta con enoxaparina sodica 4000 UI (Clexane, Sanofi-Aventis SpA), iniziata la sera prima e proseguita per 35 giorni dopo l'intervento.

Tutti gli interventi sono stati eseguiti da un unico operatore (P.R.) che ha utilizzato la medesima tecnica chirurgica e la stessa protesi cementata (P.F.C. Sigma, Depuy-Johnson\&Johnson) in tutti i pazienti. L'uso di acido tranexamico non è stato consentito.

Ad inizio intervento prima dell'incisione è stato posizionato un laccio emostatico alla radice dell'arto gonfiato. Dopo la cementazione della protesi, il campo chirurgico è stato accuratamente lavato con soluzione fisiologica per rimuovere i detriti dai tessuti molli, e, solo a questo punto, è stato rilasciato il tourniquet, in modo da poter iniziare l'emostasi primaria tramite elettrocauterizzazione. Dopo queste fasi, nel gruppo di trattamento sono stati nebulizzati $5 \mathrm{ml} \mathrm{di}$ Evicel $^{\circledR}$ sui tessuti molli a distanza di $10-15 \mathrm{~cm}$, prima a $90^{\circ}$ di flessione, poi in estensione, ponendo particolare attenzione alla regione posteriore dell'articolazione e al tessuto sottocutaneo.

Dopo l'applicazione del farmaco o dopo l'emostasi primaria, in caso del gruppo di trattamento o di controllo rispettivamente, l'accesso è stato suturato e sono stati posizionati due drenaggi in aspirazione, uno intra-articolare e l'altro sottocutaneo, rimossi il giorno dopo senza mai reinfonderne il contenuto al paziente. Dopo l'intervento il ginocchio è stato mantenuto in estensione con un bendaggio vascolare dell'arto.

Nel post-operatorio valori di $\mathrm{Hb}$ inferiori a $8 \mathrm{~g} / \mathrm{dl}$ impongono di far assoluto ricorso alla trasfusione sanguigna, mentre con valori maggiori di $\mathrm{Hb}$ la decisione di sottoporre un paziente a trasfusione dipende dalle condizioni cliniche del paziente e dal suo livello di tolleranza all'anemia.

L'outcome primario dello studio per valutare l'effetto del farmaco è stato il livello di Hb nei primi 7 giorni post-operatori. I livelli di $\mathrm{Hb}$ e ematocrito $(\mathrm{Ht})$ sono stati registrati $1 \mathrm{~h}, 12 \mathrm{~h}, 1,2$, 3, e 7 giorni post-intervento, così come la quantità di sangue nei drenaggi e il numero di trasfusioni. 


\section{Raccolta dati}

La quantità di globuli rossi persa è stata calcolata con la formula di Mercuriali e Inghilleri [4] basata sul valore di ematocrito del paziente.

$$
\mathrm{RBC} \text { loss }=\mathrm{V}_{0} \times\left(\mathrm{Ht}_{\text {pre-op }}-\mathrm{Ht}_{7 \mathrm{gg} \text { post-op }}\right)+\mathrm{RBC} \text { trasfusi }
$$

$\mathrm{RBC}$ (red blood cells) loss = quantità di eritrociti persi

$\mathrm{V}_{0}=$ volume totale di sangue presunto

$\mathrm{RBC}$ transfusi $=$ eritrociti trasfusi con trasfusioni omologhe

Vo è stato calcolato secondo la formula di Nadler [5]:

$$
\begin{aligned}
& \mathrm{V}_{0} \text { uomo: } 0,3669 \times \text { altezza }(\mathrm{m})^{3}+0,03219 \times \text { peso }(\mathrm{Kg})+0,6041 \\
& \mathrm{~V}_{0} \text { donna: } 0,3561 \times \text { altezza }(\mathrm{m})^{3}+0,0338 \times \text { peso }(\mathrm{Kg})+0,1833
\end{aligned}
$$

Sempre utilizzando la precedente formula, per ogni unità di sangue omologo sono stati considerati $180 \mathrm{ml}$ di globuli rossi.

La perdita totale di sangue è stata poi calcolata attraverso la formula di Rosencher [6]:

$$
\text { Blood Loss }=\text { RBC loss } / 0,35
$$

$$
0,35=\text { valore di ematocrito minimo da raggiungere }
$$

\section{Analisi statistiche}

Le analisi statistiche sono state condotte al termine della raccolta dati e sono state effettuate con un software statistico (SPSS for Windows versione 17).

Il calcolo della power analysis ha dimostrato che, con una potenza dell' $80 \%$ e un livello di significatività del 5\% in un campione con almeno 30 pazienti per gruppo, la differenza tra i valori medi di $\mathrm{Hb}$ doveva essere di almeno $1 \mathrm{~g} / \mathrm{dl}$.

Il t-test di Student per campioni indipendenti a doppia coda è stato utilizzato per valutare differenze significative tra valori medi di $\mathrm{Hb}$, ematocrito e perdite peri-operatorie dei due gruppi.

Il test non parametrico di Mann-Whitney (U test) è stato utilizzato per il confronto delle variabili con distribuzione dei valori non di tipo Gaussiano. 
Le variabili dicotomiche sono state analizzate mediante il test Chi Square o Fisher's Exact test. Il Chi Square test for trend è stato utilizzato per la valutazione dell'associazione tra il tasso di trasfusione omologa e i valori crescenti di $\mathrm{Hb}$ pre-operatori.

Tutti i p-value riportati sono stati a doppia coda, con livello di significatività determinato da un $\mathrm{p}<0,05$.

\section{RISULTATI}

Tra Febbraio 2011 e Aprile 2012 sono stati reclutati 62 pazienti sui 95 esaminati: 31 sono stati trattati con Evicel ${ }^{\circledR}$ (gruppo di trattamento) e 31 sono stati inclusi nel gruppo di controllo.

Il gruppo di controllo era costituito da 22 donne (71\%) e 9 uomini (29\%) mentre quello di trattamento da 26 donne $(83,9 \%)$ e 5 uomini $(16,1 \%)$. La differenza tra i due gruppi non era significativa $(\mathrm{p}>0,05)$.

L'età media dei pazienti del gruppo di controllo non era significativamente differente da quella del gruppo di controllo ( 71 vs $69 ; \mathrm{p}>0,05)$.

I due gruppi erano inoltre paragonabili per Body Mass Index (BMI), sesso, volume ematico totale, $\mathrm{Hb}$ pre-operatoria, American Society Anestesiologists (ASA) score e tempo di laccio (Tabella 1).

\section{Efficacia del trattamento}

Il tasso di trasfusione è stato del 32,3\% nel gruppo di controllo e del 25,8\% in quello di trattamento (p-value=0,5). La perdita totale media era di 1,9 1 nel gruppo di controllo e di 1,8 1 in quello di trattamento ( $p$-value $=0,5)$. Le perdite nei drenaggi non erano significativamente differenti nei due gruppi (gruppo di trattamento: $601 \mathrm{ml}$; gruppo di controllo: $549 \mathrm{ml}$; p-value=0,4). Le perdite in termini di RBC erano inferiori nel gruppo di trattamento ma la differenza non era statisticamente significativa (622 $\mathrm{ml}$ contro $664 \mathrm{ml}, \mathrm{p}$-value=0,4) (Tabella 2).

Come si evidenzia nella Figura 1 non è stata osservata alcuna differenza tra i due gruppi per i valori di $\mathrm{Hb}$ nei giorni successivi all'intervento.

\section{Gruppo di trattamento}

L'analisi dei pazienti del gruppo di trattamento ha mostrato una differenza significativa in termini di Hb pre-op (Tabella 3 ) a favore dei pazienti che non sono stati sottoposti a trasfusione omologa $(12,4$ $\mathrm{g} / \mathrm{dl}$ per i pazienti che sono stati trasfusi; 13,6 per gli altri non trasfusi; $\mathrm{p}$-value $=0,01$ ).

Le perdite ematiche totali e il volume di globuli rossi erano significativamente più alte nei pazienti sottoposti a trasfusione (perdite totali: 2,2 1 contro 1,7 1; p-value=0,01; volume di globuli rossi perso: $743,8 \mathrm{ml}$ contro $580 \mathrm{ml}$; p-value $=0,02$ ). 
Nessuna differenza è stata osservata in termini di $\mathrm{Hb}$ persa nei due gruppi (pazienti trasfusi: $218 \mathrm{~g}$; pazienti non trasfusi: $193 \mathrm{~g}$; $\mathrm{p}$-value $=0,2$ ).

L'andamento nel tempo della concentrazione di $\mathrm{Hb}$ evidenzia valori significativamente superiori fino al secondo giorno post-operatorio nel gruppo di pazienti non trasfusi. Tale concentrazione non presenta differenze nei due gruppi sperimentali a 3 e 7 giorni dopo l'intervento (Figura 2).

\section{Gruppo di controllo}

Nel gruppo di controllo i valori pre-operatori di $\mathrm{Hb}$ non erano significativamente inferiori nei pazienti sottoposti a trasfusione rispetto ai pazienti non trasfusi $(13,1 \mathrm{~g} / \mathrm{dl}$ nei pazienti trasfusi contro 13,6 g/dl nei pazienti non trasfusi; $\mathrm{p}$-value=0,2). I globuli rossi totali persi, Hb totale persa e il volume totale perso invece presentavano valori significativamente superiori nei pazienti trasfusi (Tabella 4).

L'andamento nel tempo della concentrazione di $\mathrm{Hb}$ evidenzia valori significativamente superiori fino a 7 giorni dopo l'intervento (Figura 3).

\section{Analisi dell'incidenza di trasfusione}

Il tasso di trasfusione nel gruppo di trattamento è risultato significativamente associato al valore di $\mathrm{Hb}$ pre-operatoria $(\mathrm{p}<0,05)$. Questo valore si riduceva significativamente in modo lineare all'aumentare dei valori pre-operatori di $\mathrm{Hb}(\mathrm{p}=0,009$ Chi Square test for trend, Figura 4).

Nel gruppo di controllo, il tasso di trasfusione non era significativamente associato al valore di $\mathrm{Hb}$ pre-operatoria $(\mathrm{p}=0,2)$ (Figura 4$)$.

Analizzando il sottogruppo di pazienti con $\mathrm{Hb}$ pre-operatoria $>13,5 \mathrm{~g} / \mathrm{dl}$ la percentuale di trasfusione omologa nel gruppo di trattamento era di $5 \%(1 / 20)$, mentre in quello di controllo era di $25 \%(5 / 20)(p=0,1 ; 1-\beta=46 \%)$.

\section{DISCUSSIONE}

I risultati del nostro studio non mostrano significativi vantaggi nell'utilizzo di Evicel ${ }^{\circledR}$ (Johnson \& Johnson Wound Managment, Ethicon, Somerville, NJ) in termini di riduzione di perdite ematiche, $\mathrm{Hb}$ e richiesta di trasfusione. Tuttavia nel gruppo di trattamento abbiamo osservato una riduzione dell'incidenza di trasfusione all'aumentare dei livelli di $\mathrm{Hb}$ pre-operatori.

Molti studi in letteratura hanno valutato l'efficacia delle colle di fibrina nell'artroprotesi totale di ginocchio. Le differenze nei diversi disegni sperimentali quali quantità di farmaco utilizzato $(5,10$ o $20 \mathrm{ml}$ ), utilizzo del laccio emostatico (rilascio prima o dopo dell'impianto protesico), inserimento 
dei drenaggi post-operatori (in aspirazione o meno, per 24 o 72 ore post-operatorie) rendono però difficile trarre conclusioni univoche sul loro utilizzo [7-9]. Inoltre, tra i molti studi già pubblicati una sostanziale differenza risiede nel farmaco utilizzato. Infatti, Bernasek e colleghi [8] risultano gli unici autori, a nostra conoscenza, ad aver precedentemente utilizzato Evicel ${ }^{\circledR}$; gli altri studi hanno invece utilizzato Quixil, che oltre ad essere costituito da fibrinogeno e trombina umana, contiene anche acido tranexamico, dimostratosi efficace nella riduzione dei sanguinamenti anche quando usato singolarmente [10].

Nel nostro studio, per evitare ogni possibile bias in modo da uniformare il più possibile il trattamento dei pazienti, abbiamo impiegato le seguenti strategie: 1) non far pre-depositare ai pazienti unità di sangue autologhe alla banca del sangue; 2) non re-infondere il sangue presente all'interno dei drenaggi; 3) evitare l'uso intra-operatorio di acido tranexamico; 4) utilizzare la stessa tecnica chirurgica e il medesimo impianto in tutti i pazienti operati.

Utilizzando solo strategie intra-operatorie per il controllo del sanguinamento (tourniquet fino a prima di un accurata emostasi), non abbiamo osservato alcuna differenza tra $\mathrm{i}$ due gruppi sperimentali in termini di perdite ematiche.

Analizzando poi il gruppo di trattamento, abbiamo riscontrato una relazione lineare tra il tasso di trasfusione e i valori di $\mathrm{Hb}$ pre-operatoria. Nel gruppo di trattamento abbiamo osservato una percentuale di trasfusione del 5\% nei pazienti con valori di $\mathrm{Hb}$ pre-operatoria $>13,5 \mathrm{~g} / \mathrm{dl}$ contro il $25 \%$ del controllo. Possiamo quindi ipotizzare che i pazienti con un livello di $\mathrm{Hb}$ pre-operatorio maggiore di 13,5 g/dl possano essere avvantaggiati dall'utilizzo di Evicel ${ }^{\circledR}$ in termini di riduzione della richiesta di trasfusione. La differenza osservata tra i due gruppi di studio non risulta però significativa, sarebbe quindi necessario aumentare la numerosità campionaria ad almeno 40 pazienti per gruppo per stabilire la validità dei risultati con una potenza almeno dell' $80 \%$ ed affermare con sicurezza l'efficacia del trattamento.

In conclusione, $\mathrm{i}$ dati presenti in letteratura ed i risultati ottenuti dal nostro studio mostrano che 1'utilizzo di Evicel ${ }^{\circledR}$ come adesivo tissutale per ridurre le perdite ematiche intra- e post-operatorie, non riporta benefici apprezzabili per il paziente ai dosaggi finora testati. Ulteriori studi saranno necessari per stabilire il valore teraperutico/l'efficacia delle colle di fibrina a dosaggi superiori a quelli finora sperimentati. 
Tabella 1:

\begin{tabular}{|c|c|c|c|}
\hline Età & $71( \pm 6,5)$ & $69( \pm 8)$ & p-value \\
\hline BMI & $29( \pm 3,7)$ & $28( \pm 5,6)$ & n.s. \\
\hline $\begin{array}{c}\text { \% Donne } \\
\text { Volume } \\
\text { ematico(I) }\end{array}$ & $41 \%$ & $84 \%$ & n.s. \\
\hline $\begin{array}{c}\text { Hb preop } \\
\text { (g/dL) }\end{array}$ & $13,4( \pm 0,7)$ & $4,3( \pm 0,7)$ & n.s. \\
\hline $\begin{array}{c}\text { ASA score } \\
\text { Tempo di }\end{array}$ & $1,9( \pm 0,4)$ & $13,2( \pm 0,9)$ & n.s. \\
laccio (min) & $54( \pm 9,7)$ & $55( \pm 9,5)$ & n.s. \\
\hline
\end{tabular}


Tabella 2:

\begin{tabular}{|c|c|c|c|c|}
\hline & Drenaggio (ml) & RBC persi (ml) & $\begin{array}{c}\text { Perdita totale di } \\
\text { sangue (l) }\end{array}$ & $\begin{array}{c}\text { Tasso di } \\
\text { trasfusione }\end{array}$ \\
\hline Gruppo Controllo & $549( \pm 269)$ & $664( \pm 226)$ & $1,9 \pm(0,7)$ & $32,3 \%(10 / 31)$ \\
\hline Gruppo Trattamento & $601( \pm 250)$ & $622( \pm 175)$ & $1,8( \pm 0,5)$ & $25,8 \%(8 / 31)$ \\
\hline p-value & 0,4 & 0,4 & 0,5 & 0,5 \\
\hline
\end{tabular}


Tabella 3:

\section{Gruppo Trattamento}

\begin{tabular}{|c|c|c|c|c|}
\hline & $\begin{array}{c}\text { Hb preop } \\
\mathbf{( g / d l )}\end{array}$ & $\begin{array}{c}\text { RBC persi } \\
(\mathbf{m l})\end{array}$ & $\begin{array}{c}\text { Hb persa } \\
\mathbf{( g )}\end{array}$ & $\begin{array}{c}\text { Perdita } \\
\text { totale di } \\
\text { sangue (I) }\end{array}$ \\
\hline Trasfusione & $12,4( \pm 0,7)$ & $743,8( \pm 202)$ & $218( \pm 64,7)$ & $2,2(0,6)$ \\
\hline No trasfusione & $13,6( \pm 0,8)$ & $580( \pm 146)$ & $193( \pm 50)$ & $1,7(0,4)$ \\
\hline p-value & $\mathbf{0 , 0 1}$ & $\mathbf{0 , 0 2}$ & 0,2 & $\mathbf{0 , 0 1}$ \\
\hline
\end{tabular}


Tabella 4:

\begin{tabular}{|c|c|c|c|c|}
\hline \multicolumn{5}{|c|}{ Gruppo Controllo } \\
\hline & $\begin{array}{c}\text { Hb preop } \\
\text { (g/dl) }\end{array}$ & RBC loss (ml) & $\begin{array}{c}\text { Hb persa } \\
\mathbf{( g )}\end{array}$ & $\begin{array}{c}\text { Perdita } \\
\text { totale } \\
\text { sangue (I) }\end{array}$ \\
\hline Trasfusione & $13,1( \pm 0,7)$ & $862( \pm 224)$ & $269( \pm 73)$ & $2,6( \pm 0,75)$ \\
\hline No trasfusione & $13,6( \pm 0,9)$ & $570( \pm 158)$ & $188( \pm 53)$ & $1,6( \pm 0,5)$ \\
\hline p-value & 0,2 & $<\mathbf{0 , 0 0 1}$ & $\mathbf{0 , 0 0 2}$ & $<0,001$ \\
\hline
\end{tabular}


Figura 1:

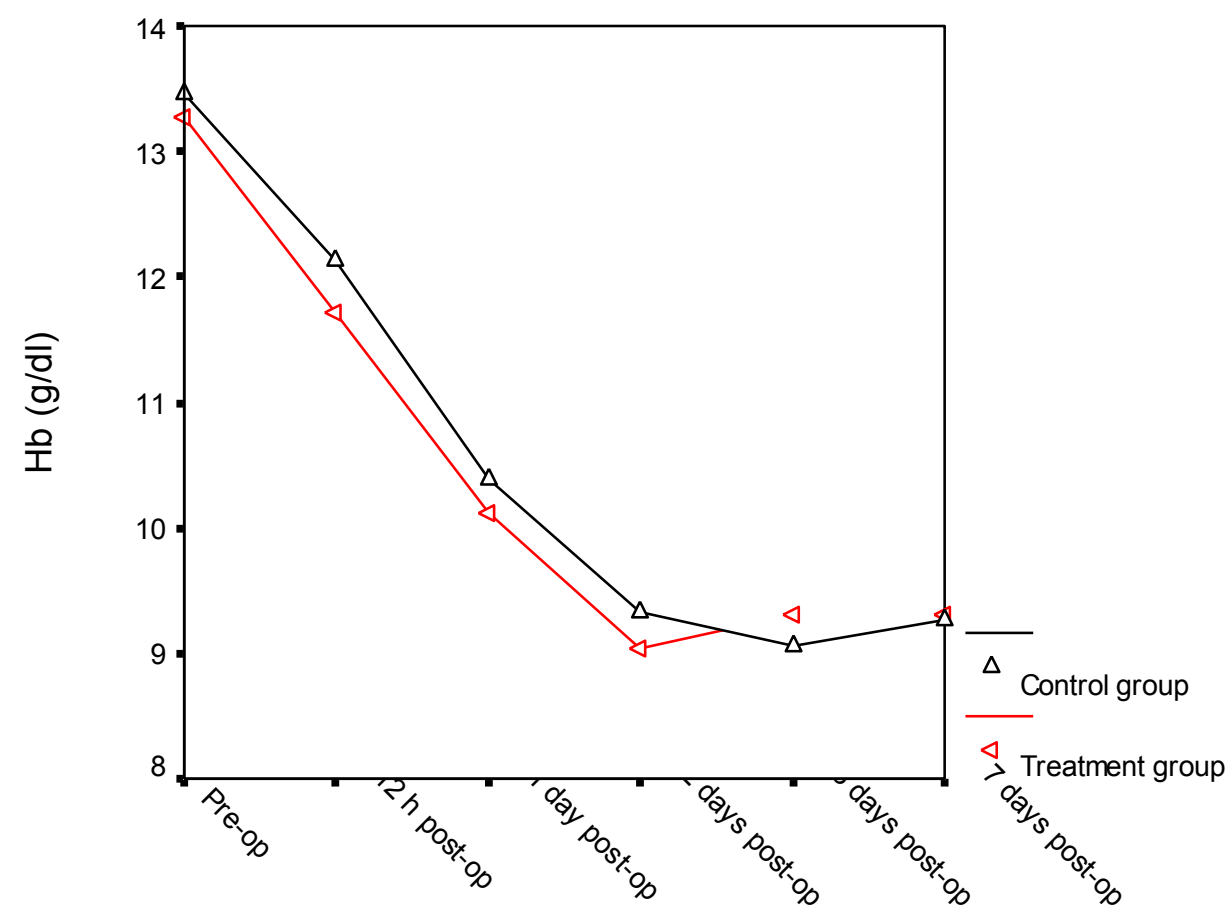


Figura 2:

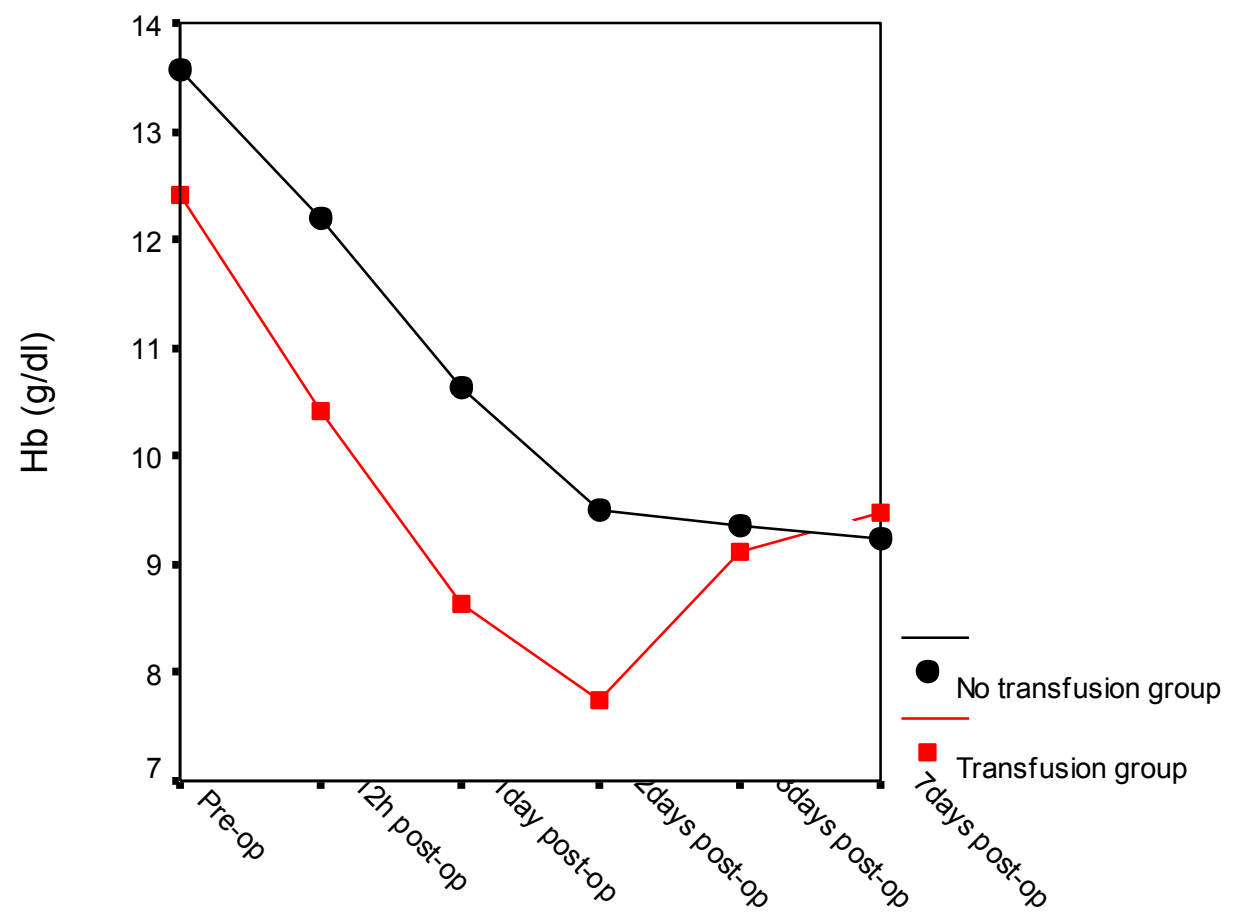


Figura 3:

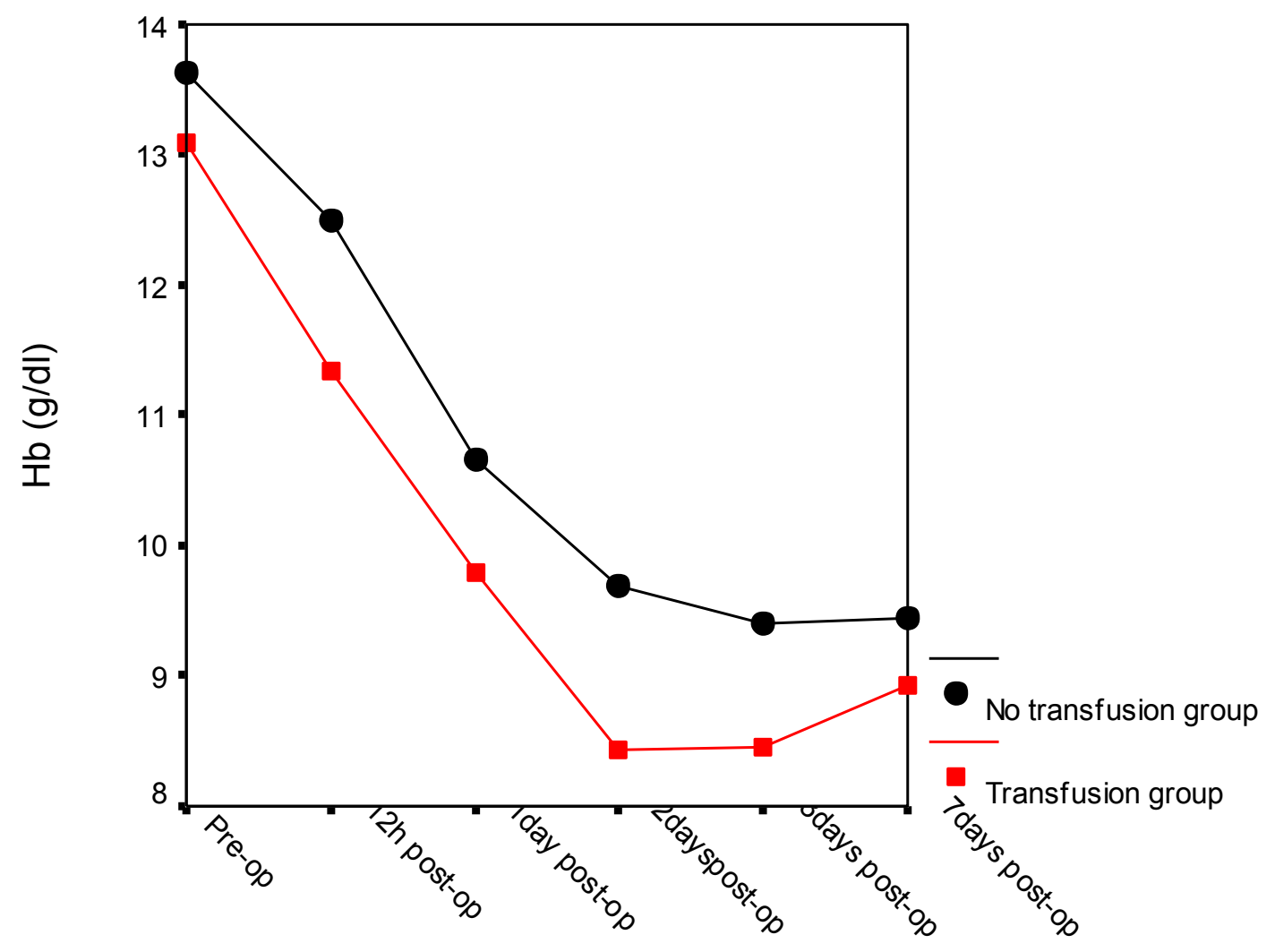


Figura 4:

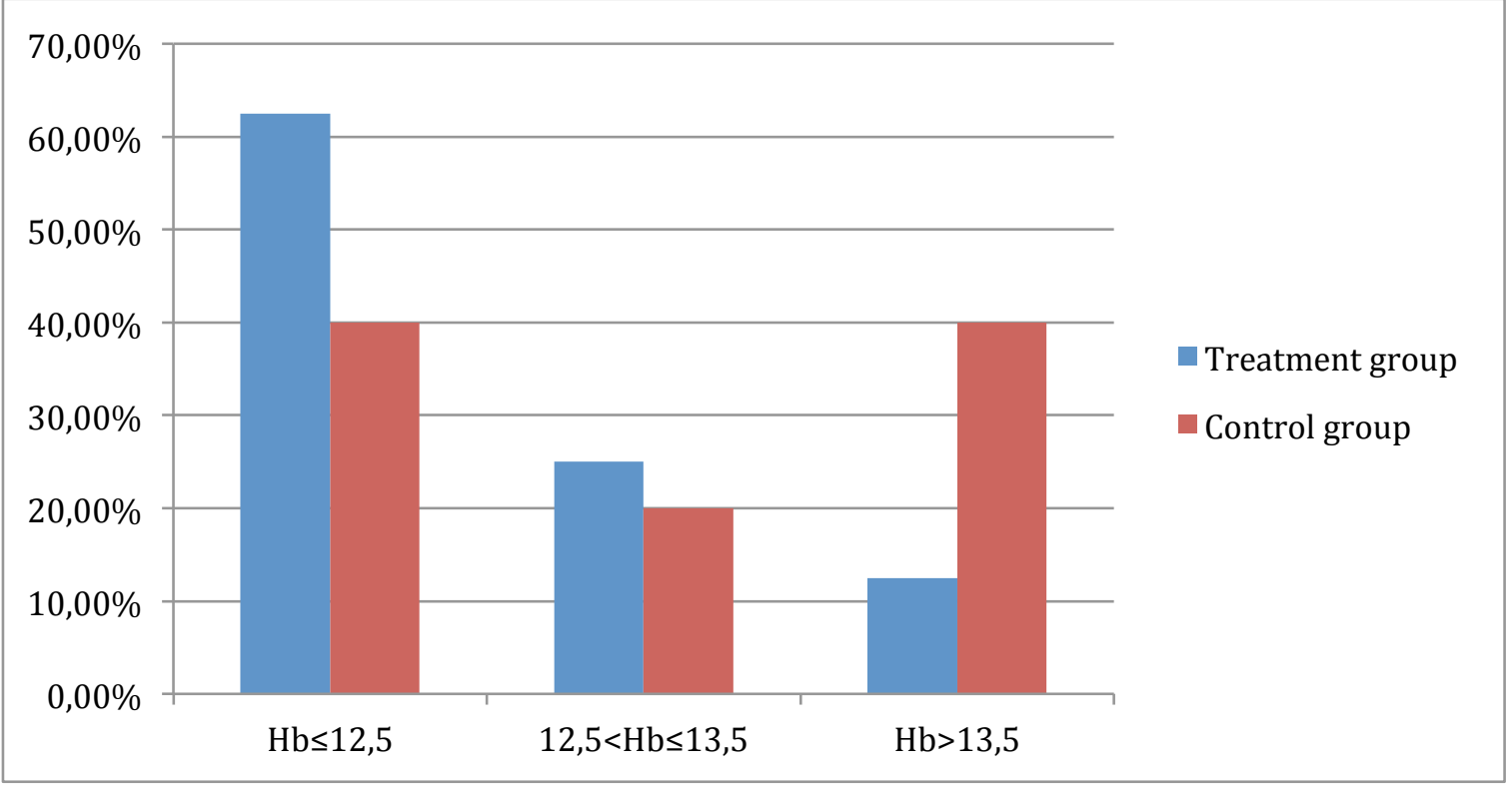




\section{BIBLIOGRAFIA}

1 Lotke P, Faralli VJ, Orenstein E, Ecker M. Blood loss after total knee replacement. In: The Journal of bone and joint surgery; August 1991:1036-40.

2 Sehat K, Evans R, Newman J. How much blood is really lost in total knee arthroplasty: correct blood management should take hidden loss into account. In: Knee; 2000:151-5. 3 Wong J, Abrishami A, El Beheiry H, et al.

Topical application of tranexamic acid reduces postoperative blood loss in total knee arthroplasty: a randomized, controlled trial. In: J Bone Joint Surg Am; 2010:2503-13.

4 Mercuriali F, Inghilleri G. Proposal of an algorithm to help the choice of best transfusion strategy. In: Current medical research and opinion; 1996:465-78.

5 Nadler S, Hidalgo JH, Bloch T. Prediction of blood volume in normal human adults. 1962;51:224-32.

6 Rosencher N, Kerkkamp HEM, Macheras G, et al.

Orthopedic Surgery Transfusion Hemoglobin European Overview (OSTHEO)

study: blood management in elective knee and hip arthroplasty inEurope. In: Transfusion 2003:45969.

7 Levy O, Martinowitz U, Oran A, Tauber C, Horoszowski H.

The use of fibrin tissue adhesive to reduce blood loss and the need for blood transfusion after total knee arthroplasty. A prospective, randomized, multicenter study. In: J Bone Joint Surg Am 1999:1580-8.

8 Bernasek TL, Burris RB, Fujii H, Levering MF, Polikandriotis JA, Patterson JJ. Effect on Blood Loss and Cost-Effectiveness of Pain Cocktails, Platelet-Rich Plasma, or Fibrin Sealant After Total Knee Arthroplasty. In: The Journal of arthroplasty; 2012:1448 -51.

9 Kluba T, Fiedler K, Kunze B, Ipach I, Suckel A. Fibrin sealants in orthopaedic surgery: practical experiences derived from use of QUIXIL $®$ in total knee arthroplasty. In: Arch Orthop Trauma Surg 2012:1147-52.

10 Alshryda S, Sarda P, Sukeik M, Nargol A, Blenkinsopp J, Mason JM.

Tranexamic acid in total knee replacement: a systematic review and meta-analysis. In: J Bone Joint Surg Br 2011:1577-85. 
Norme etiche:

Lo studio è stato condotto conformemente alle linee guida del comitato etico dell'ASL di Milano 2 (18 febbraio 2011, protocollo numero 2603), e quindi conformemente alle norme etiche stabilite dalla Dichiarazione di Helsinki del 1964. I pazienti reclutati per lo studio hanno fornito il loro previo consenso. 